\title{
SETE CRÍTICAS À SALA DE AULA INVERTIDA
}

\author{
Marcelo Valério ${ }^{1}$ \\ Ana Lúcia Olivo Rosas Moreira²
}

\section{RESUMO}

A sala de aula invertida consiste de um arranjo didático no qual os estudantes têm contato prévio com os conteúdos e dedicam o tempo em classe para tarefas de operacionalização e aplicação dos conhecimentos. Por meio de materiais produzidos e/ou disponibilizados antecipadamente pelos professores, quase sempre com mediação de tecnologias digitais, abrevia-se a tradicional aula expositiva e focaliza-se o encontro presencial em metodologias de aprendizagem ativa. 0 número de experiências e pesquisas sobre a sala de aula invertida vem crescendo, mas a precedência e a extrapolação dos resultados positivos tornam questionável a rápida acolhida acadêmica e midiática do modelo. Derivado de uma ampla revisão bibliográfica, este ensaio objetiva compilar e repercutir visões teóricas e resultados empíricos críticos à sala de aula invertida. São apresentadas sete teses que ilustram a necessidade de atenção, aprofundamento e parcimônia quando da adoção e defesa dessa estratégia por pesquisadores, professores e instituições de ensino.

Palavras-chave: Inovação pedagógica. Metodologias ativas. Ensino centrado no estudante. Ensino híbrido.

\section{SEVEN CRITICISMS OF FLIPPED CLASSROOM}

\section{ABSTRACT}

In a flipped classroom, students have a previous contact with the lessons materials and dedicate in-class time to the application of the knowledge. These materials are produced and/or made available by teachers and faculty members with the mediation of digital technologies. Thereby, traditional lectures are supressed and active learning methodologies take place. The number of experiences and research about flipped classroom has been increasing, but the extrapolation of early positive results makes the acceptance of the model, by the academia and the media, questionable. In this article we systematize theoretical perspectives and empirical data from a bibliographical review, aiming to discuss about critical results of the flipped classroom. We present seven topics that reflect the need for attention and parsimony of researchers, educators and institutions, before the defence and adoption of this didactical approach.

Keywords: Pedagogical innovation. Active learning. Student-centered methods. Blended learning.

RECEBIDO EM: $15 / 4 / 2018$

ACEITO EM: 9/5/2018

\footnotetext{
${ }^{1}$ Docente da Universidade Federal do Paraná (UFPR), campus avançado Jandaia do Sul. Mestre em Educação Científica e Tecnológica (UFSC) e doutorando em Educação para a Ciência (UEM). marcelovalerio@ufpr.br

${ }^{2}$ Docente da Universidade Estadual de Maringá (UEM). Mestre em Botânica (UFPR) e doutora em Ciências (Ufscar). alormoreira@uem.br
} 


\section{SITUANDO E CARACTERIZANDO O FENÔMENO}

Em suas casas, estudantes leem um capítulo de um livro-texto, assistem a duas videoaulas e ouvem um podcast sobre um determinado conteúdo de ensino. Tudo planejado, compartilhado e acompanhado em uma plataforma digital pelo professor. Ao chegar em sala, são desafiados a responder rapidamente a um quizz sobre o assunto estudado. O professor analisa as respostas e propõe que os alunos as debatam com colegas em pequenos grupos. Enquanto isso, circula e acompanha a desenvoltura e o desempenho na tarefa, concedendo orientações e feedbacks aos estudantes. Identifica uma dúvida recorrente, pede a atenção e explana, brevemente, a respeito.

De modo exemplar, é assim que funciona uma sala de aula invertida (SAI), um arranjo didático em que o tempo fora de sala é dedicado ao estudo prévio e o tempo em sala às atividades de operacionalização e aplicação.

Recentemente, a SAI se tornou um fenômeno acadêmico e midiático, assumindo rótulos de revolução e inovação educacional. Seu caráter disruptivo iniciaria na Pedagogia, com o rompimento da tradição, a negação do ensino transmissivo e a crítica à passividade do estudante no processo ensino-aprendizagem. Nesse sentido, a proposta costuma ser filiada aos conceitos de "aprendizagem ativa" e do "ensino centrado no estudante" (MICHAEL, 2006; MASCOLO, 2009), e metodologicamente opera por estratégias como a "instrução por pares" (MAZUR, 1997; LYMAN, 1987), a "aprendizagem baseada em problemas" (BARROWS; TAMBLYN, 1980), os "grupos de trabalho colaborativo" (MICHAELSEN; KNIGHT; FINK, 2004), o "ensino imediato" (NOVAK et al., 1999) e a "aprendizagem por investigação" (CARVALHO, 2013), entre outras "metodologias ativas" (MICHAEL, 2006; MASCOLO, 2009; BERBEL, 2011; ROCHA; LEMOS, 2014).

A segunda via da revolução estaria no papel central das tecnologias digitais nas relações estudantes-conhecimentos-professores (BISHOP; VERLEGER, 2013; MOFFETT, 2015). Em um mundo de tecnologias digitais ubíquas e no qual a educação formal se revela anacrônica, a SAI tem sido frequentemente apresentada como uma modalidade e-learning e como um caminho rumo à educação híbrida (ou blended learning) - ora com o processo ocorrendo on-line, ora presencialmente (VALENTE, 2014). Nesse sentido, foi apresentado um grande número de ferramentas durante os últimos anos para a produção, disponibilização e desenvolvimento de conteúdos educacionais, bem como para a experiência direta em sala e para a avaliação dos estudantes. ${ }^{3}$ Softwares de gravação e publicação de videoaulas, sistemas de resposta de audiência (clickers), recursos vinculados às mídias sociais e plataformas virtuais de aprendizagem, são apenas alguns exemplos do instrumental disponível e já em uso.

O interesse acadêmico foi crescente nas últimas duas décadas, expandindo rapidamente o volume de publicações e referências em diferentes graus de ensino, áreas de conhecimentos e contextos educativos, por todo o mundo e nos mais diversos veículos. O maior volume de dados vem de áreas de intenso apelo tecnológico, como as Ciências

3 O Grupo Planeta, empresa espanhola do mercado editorial, publicou, em seu portal "AulaPlaneta", um infográfico com aproximadamente 40 ferramentas distintas associadas à sala de aula invertida. Disponível em: <http://www. aulaplaneta.com/2015/05/12/recursos-tic/40-herramientas-para-aplicar-la-metodologia-flipped-classroom-en-elaula-infografia/>. 
da Saúde e as Engenharias, ou onde impera a tradição pedagógica expositiva, como as Ciências Exatas (GIANNAKOS; KROGSTIE; CHRISOCHOIDES, 2014; ZAINUDDIN; HALILI, 2016). Os resultados são mesmo promissores, e registram haver interações mais frequentes e producentes entre professores e alunos e entre os próprios alunos (DESLAURIERS; SCHELEW; WIEMAN, 2011; BERGMANN; SAMS, 2012; BROWN, 2012; STRAYER, 2012; DRISCOLL, 2012; GALINDO; QUINTANA, 2016; OFUGI, 2016); maior oportunidade de trabalho individualizado, adequado ao ritmo dos alunos (LAGE; PLATT; TREGLIA, 2000; DRISCOLL, 2012; BROWN, 2012; JOHNSON, 2013); incremento em habilidades comunicativas, de pensamento crítico e resolução de problemas (GÓMEZ, 2016; TURAN; GOKTAS, 2016; TAZIJAN et al., 2017); e melhora significativa no clima de aprendizagem, desempenho e frequência dos estudantes (MAZUR, 2009; PAPADOPOULOS; ROMAN, 2010; TREVELIN; PEREIRA; OLIVEIRA NETO, 2013; NOURI, 2016; OLAKANMI, 2016; BUSATO et al., 2016; SENGEL, 2016; KIM; KIM, 2017; DESLAURIERS; SCHELEW; WIEMAN, 2011; MARLOWE, 2012).

Logo, uma série de guias e manuais de apresentação do modelo SAI começaram a ser produzidas por instituições específicas, consórcios institucionais e pelo mercado de mídias digitais na primeira década dos anos 2000. Segundo Bart (2013), uma pesquisa com as universidades estadunidenses dava conta de que, à época, 29\% dessas instituições já estariam introduzindo o modelo e que outros $27 \%$ teriam planos de fazê-lo em breve.

As expressões flipped classroom e inverted classroom também despertaram rapidamente o interesse midiático: desde 2012, periódicos gerais como o The New York Times (FITZPATRICK, 2012) e especializados, como o Chronicle of Higher Education (BERRETT, 2012; MANGAN, 2013; NESHYBA, 2013; TALBERT, 2014; YOUNG, 2015), repercutem as experiências de dezenas de universidades nos Estados Unidos e no Canadá. Valente (2014) sugere que essas publicações impulsionaram a SAI como um novo chavão da educação contemporânea. E essa tendência se confirmou em 2014 no NMC Horizon Report, um prestigiado relatório que identifica e descreve desenvolvimentos e tecnologias emergentes com potencial de impacto no âmbito do Ensino Superior (JOHNSON et al., 2014).

No Brasil, materiais institucionais também começaram a conceder espaço para o assunto (FUNDAÇÃO..., 2015) e vários autores discorreram em defesa das tecnologias e das metodologias ativas como indutoras dos currículos e de novas práticas de ensino (MORAN, 2015; CASTELLO BRANCO et al., 2016). Além disso, um grupo de 40 instituições de Ensino Superior brasileiras, públicas e privadas, consorciou-se às universidades estrangeiras e passou a discutir, fomentar e propagar o modelo nos últimos anos. ${ }^{4}$ Jornais de grande circulação, como O Globo (AVELLAR, 2013), Folha de São Paulo (MAIA, 2016) e Gazeta do Povo (PIVA, 2016), pautaram a SAI. Blogs de divulgação em Educação, como o Porvir (MORENA COSTA, 2016), e revistas como a Veja (BIBANO, 2014) e a Carta Capital (VILLAS-BÔAS, 2017), também se ocuparam do tema. Recentemente, a

\footnotetext{
${ }^{4}$ Ver <http://sthembrasil.com/site/> para conhecer sobre o consórcio entre Instituições de Ensino Superior brasileiras e a Laspau, descrita como uma iniciativa para o desenvolvimento da inovação acadêmica por meio do "Programa Acadêmico e Profissional para as Américas", afiliado à Universidade de Harvard. 
revista mais lida entre os profissionais de educação brasileiros estampou a SAI em matéria de capa em um número que orientava como utilizar as mais promissoras inovações educacionais (INOVAÇÃO..., 2017).

Muito rapidamente, o modelo SAI foi acolhido pela comunidade pedagógica e ocupou um lugar de destaque nos discursos e nas práticas educacionais contemporâneas. Ainda assim, não deixam de permanecer controversas as suas origens, os seus fundamentos pedagógicos, a tradição de pesquisa que sustenta seus resultados, e mesmo as implicações de sua adoção nos diferentes contextos educativos. Não se trata de negar a profusão de resultados positivos, mas, também, de repercutir seus contrapontos e valorizar o debate acadêmico. É cabível que a comunidade educativa se proponha a analisar atentamente as transformações que o modelo SAI propõe sobre os fins e meios da educação formal; afinal, quem quer que se dedique a revisar a história da educação reconhecerá muitos exemplos de pretensas transformações paradigmáticas que esmoreceram diante da realidade.

\section{ENCAMINHAMENTOS METODOLÓGICOS}

Resultado do acompanhamento da literatura sobre o tema entre os anos de 2014 e 2017, este ensaio apresenta uma compilação das visões teóricas e resultados empíricos críticos à sala de aula invertida. Trata-se, portanto, de uma argumentação sem pretensões sistemáticas, mas que se vê sustentada em ampla revisão bibliográfica.

Durante o período citado, todas as referências on-line aos marcadores "sala de aula invertida", "flipped classroom" e "inverted classroom", foram localizados e alertados por meio da ferramenta de pesquisa Google Scholar. Títulos, resumos e palavras-chave dos artigos (em português, inglês e espanhol) foram analisados. Quando indicavam algum aspecto crítico à SAI foram acessados e lidos na íntegra. Todas as produções, descritas como revisões de literatura sobre a temática, também foram estudadas a pleno. Esse corpus se ampliou, ainda, com a identificação e a busca ativa de referências cruzadas durante as leituras.

O resultado foi um conjunto de críticas à SAl, que vai desde interpretações teóricas até juízos empíricos. A compilação, síntese e interpretação teórica dos argumentos listados culminou nas sete críticas apresentadas adiante. Entende-se que elas ilustram a necessidade de atenção, aprofundamento e parcimônia quando da adoção e defesa dessa estratégia por pesquisadores, professores e instituições de ensino.

\section{Crítica 1: Crise de identidade}

O mérito acadêmico de qualquer proposição, teórica ou metodológica, deve ser referenciado a quem de direito. Seguindo essa premissa ética, quase todos os textos, principalmente os acadêmicos, buscam identificar os primeiros passos da SAI. A demarcação, contudo, de uma origem ou filiação para o modelo didático, é confusa e está longe de ser consensual (ABEYSEKERA; DAWSON, 2014).

Nas pesquisas acadêmicas, produzidas com mais desvelo e aprofundamento, é comum ler referências ao trabalho de Lage, Platt e Treglia (2000), quando se relata uma experiência desenvolvida em 1996 em uma disciplina de Microeconomia no Ensino Su- 
perior em Ohio, nos Estados Unidos. A publicação se apoia em estudos sobre a diversidade de ritmos e estilos de aprendizagem dos estudantes, sugerindo a SAI como um método de ensino (sic) adequado para lidar com o problema. $\mathrm{O}$ artigo analisa a percepção de estudantes e professores, valorizando resultados da adoção da SAl em comparação com a tradicional. Pela primeira vez usa-se a expressão inverted classroom.

Outro vínculo parental da SAI reside no artigo do professor de Física da Harvard University, Eric Mazur (MAZUR, 2009). Em um número da prestigiada revista Science, o autor declara breve e eloquentemente o fim da aula expositiva, pois, ao invés de ensinar falando, agora era possível ensinar questionando! Para Mazur, seus alunos deveriam apenas "ler o material antes de vir para a aula, de modo que o tempo em sala fosse dedicado às discussões, interações entre pares, e ao tempo para assimilar e pensar" (2009, p. 51). O artigo não faz menção direta a uma SAI (nos termos flipped classroom ou inverted classroom), mas tornou conhecida uma estratégia metodológica comumente associada a ela, e que o autor já desenvolvia e defendia há mais de uma década. Trata-se da instrução por pares (peer-instruction, no original em inglês), que consiste em que os alunos, tendo estudado previamente, respondam questões ou desafios propostos e, em seguida, discutam suas respostas com os colegas, compartilhando e comparando seus resultados e procedimentos (MÜLLER et al., 2017).

A referência mais explícita e sistemática à $\mathrm{SAI}$, no entanto, encontra-se no trabaIho de Bergmann e Sams (2012). Esses dois professores de Química do Ensino Médio, preocupados com alunos ausentes ou afastados das atividades presenciais, começaram, em 2007, a gravar e disponibilizar na web vídeos de suas aulas. Em seu livro intitulado "Flip Your Classroom: Reach Every Student in Every Class Every Day", a dupla narra como os vídeos transformaram suas aulas em ambientes de discussão aberta e aprendizagem ativa, guiadas pelo estudo prévio de seus alunos rumo ao pleno domínio dos conteúdos. A obra, curta e de leitura fácil, logo virou um sucesso de vendas e foi traduzida para vários idiomas, inclusive o português, repercutindo em muitos estudos acadêmicos e em quase todas as referências midiáticas à SAI.

As bases do fenômeno estão nessas três experiências didáticas, totalmente distintas no seu referencial pedagógico, na sua área de aplicação e no seu grau de ensino. Em comum, apenas o fato de que elas reuniram, de modo pragmático, elementos pedagógicos já conhecidos: o estudo prévio, o uso de tecnologias digitais e a participação ativa do estudante em classe. Não obstante, as iniciativas se justificaram por diferentes premissas pedagógicas e operaram por diversas metodologias ou técnicas de ensino, o que parece tornar inadequada a demarcação histórica e teórica da SAl como uma única abordagem ou modelo didático.

Uma lamentável decorrência dessa crise de identidade aparece quando a literatura passa a descrever a sala de aula como uma metodologia de ensino. Trata-se de um equívoco histórico e teórico, posto que as estratégias que cabem dentro do guarda-chuvas metodológico do modelo, como a já citada instrução por pares, ou a aprendizagem baseada em problemas, ou o ensino por investigação, não são necessariamente aparentados em suas bases filosóficas e pedagógicas. Ou seja, qualquer resgate histórico remeterá a um perigoso ecletismo teórico e metodológico. A identidade das ações rotuladas como SAI exige que se pondere a diversidade de contextos, áreas de aplicação, interes- 
ses de adoção, alinhamentos pedagógicos e tradições de pesquisa, pondo em dificuldades quem deseja entendê-la ou estudá-la como fenômeno educativo contemporâneo único (ABEYSEKERA; DAWSON, 2014; JENSEN; KUMMER; GODOY, 2015).

\section{Crítica 2: Não há inovações}

A filiação pedagógica da SAI, poucas vezes declarada e aprofundada na literatura sobre o tema, também prejudica sua demarcação e defesa. Acusa Pacheco (2014), que nada há de inovador nessas proposições, mas somente uma modernização de premissas ou propostas pedagógicas amplamente conhecidas e experimentadas. Para Abeysekera e Dawson (2014), trata-se de um novo rótulo para práticas antigas.

Professores em Ciências Humanas e Sociais Aplicadas há muitas décadas valem-se de estratégias de estudo prévio dirigido, seguidas pela aplicação e operacionalização conceitual em sala por meio de debates, discussões e análises. No Direito, por exemplo, o método socrático é uma tradição de ensino. Estudo prévio e aprendizagem ativa, portanto, não são disruptivos, se direcionarmos o olhar para essas outras áreas.

Mesmo o elemento da mediação tecnológica não sustenta sozinho o caráter de inovação da SAI. Em uma coluna recente, Ent (2016) destaca que o modelo SAl é uma estratégia antiga, para a qual a modernização tecnológica figura como recurso. A autora rememora o uso de tecnologias, como a fotografia, a projeção de eslaides e os arquivos de áudio no ensino, e relata a instrução antecipada, seguida por atividades práticas em contextos como o treinamento militar e a Graduação nos Estados Unidos.

O efeito modernizador da SAI viria, então, das metodologias ativas, mas tampouco elas garantem o ineditismo. É intrigante que não se encontre na literatura sobre a SAI menções à pedagogia de projetos, por exemplo. A proposta metodológica de William Kilpatrick está na essência do movimento escolanovista e carrega elementos que agora se apresentam como modernos - a saber: a atitude investigativa e o pensamento científico autônomo.

Para Bogost (2013) e Stager (2013), mesmo o arranjo didático invertido seria mais uma falácia do que uma revolução. Para esses autores, a aula expositiva continua existindo, apenas muda de lugar: se antes os estudantes assistiam a aula na escola ou universidade, agora o fazem em casa. Nas palavras de Bogost (2013, p. 1), "aula tradicional permanece viva e bem", apenas foi transformada em uma experiência de entretenimento.

\section{Crítica 3: Anarquismo pedagógico}

Existem muitos desafios para a efetivação da SAI e um deles é a familiarização da docência com o referencial pedagógico que rege o modelo. O problema é que poucos trabalhos declaram e/ou aprofundam a questão, e ninguém sabe ao certo que pressupostos sustentam o arranjo formado por estudo prévio, mediação tecnológica e metodologias ativas.

Mesmo os conceitos considerados fulcrais - a saber: aprendizagem ativa e ensino centrado no estudante - aparecem vinculados a uma miscelânea de autores e proposições teóricas costuradas de modo muito superficial. Mormente, contribuições da filosofia pragmatista [de John Dewey] e da psicologia cognitivista [de Piaget e Vygotsky] 
são reunidas sob um grande rótulo construtivista. Em sua revisão de literatura, Bishop e Verleger (2013) apresentam as conexões dessa paisagem pedagógica emergindo de referências ao "conflito cognitivo" e à "zona de desenvolvimento proximal", ampliando-se em proposições como "estilos de aprendizagem" ou "aprendizagem colaborativa", e culminando metodologicamente em práticas, como a "aprendizagem baseada em problemas".

O que se nota evidente nessas propostas é uma filiação, declarada ou não, aos ideais do movimento escolanovista, ao sugerir o foco no aluno e o aprender fazendo; a epistemologia genética de Jean Piaget, ao valorizar pedagogicamente o erro e conferir importância aos saberes prévios; o sociointeracionismo de Lev Vygostky, ao salientar o papel da interação, do diálogo, do trabalho em grupo e da mediação pedagógica; e mesmo a referenciais como a aprendizagem significativa, de Ausubel, ao propor a importância da consciência do processo de aprendizagem e da metacognição. Em suma, não há inovação no pensamento pedagógico, mas formalização e/ou estruturação de abordagens há muito defendidas e estabelecidas.

Há que se considerar, ainda, que a materialização das premissas construtivistas na sala de aula real não ocorre com facilidade. O trabalho de Tenenbaum et al. (2001) aponta justamente a dificuldade de reconhecer o construtivismo na prática de ensino direta, presencial ou a distância. Os argumentos de Matthews (2000) e Bastos Filho (2015) mostram que o construtivismo se confirma mais como jargão do que como teoria desenvolvida e estabelecida, oferecendo pouca orientação para o ensino e para a prática docente. Mascolo (2009) critica que o "ensino centrado no estudante" não valida o referencial construtivista, sobretudo porque tende a reduzir o papel da docência ao de "facilitador" ou "instrutor". No caso da SAI, tomando como exemplo a importância concedida ao estudo prévio e a recorrente menção às videoaulas, pode-se concluir que ainda se sustenta o empirismo e valida-se a tradicional transmissão do conhecimento - obviamente isso diverge de qualquer essência epistemológica construtivista.

As pesquisas sobre a SAI também costumam confundir aprendizagem com desempenho, focalizando a avaliação de aspectos cognitivos ligados à perspectiva comportamentalista e a vieses objetivistas e individualistas. $O$ recente aparecimento da taxonomia de Bloom nos referenciais de análise talvez seja um sintoma desse anarquismo pedagógico, capaz de misturar as pedagogias liberal e progressista, o tecnicismo educacional e as teorias críticas, tudo em um mesmo projeto formativo.

Em suma, não parece fazer sentido continuar advogando pela SAI enquanto houver negligência de saberes pedagógicos, ou, ainda, transigir a convivência de práticas filiadas a filosofias e pedagogias distintas.

\section{Crítica 4: Pesquisas insuficientes}

O volume e a repercussão das pesquisas sobre a SAl já produziram a necessidade e legitimaram a importância de revisões de literatura. Os trabalhos de Bishop e Verleger (2013), Giannakos, Krogstie e Chrisochoides (2014), O'Flaherty e Phillips (2015), Huber e Werner (2016) e Zainuddin e Halili (2016) são exemplares. Essas revisões de literatura permitem um olhar ampliado, pondo em debate evidências e interpretações 
do que se entende ser a tradição de pesquisa na temática. A maior parte dos resultados são positivos sim, mas decorrem de um rol de investigações carentes de solidez, rigor e amplitude (BISHOP; VERLEGER, 2013; ABEYSEKERA; DAWSON, 2014; O'FLAHERTY; PHILLIPS, 2015).

As pesquisas ainda se encontram restritas a contextos e cenários educativos específicos e singulares. Mais da metade dos trabalhos está nas áreas científicas e engenharias, e quase a totalidade dos dados publicados tem origem no contexto cultural e institucional dos Estados Unidos (GIANNAKOS; KROGSTIE; CHRISOCHOIDES, 2014; HUBER; WERNER, 2016). Os vieses culturais, portanto, marcam a pesquisa sobre a SAI e não autorizam comparações e paralelos transnacionais, tampouco a extrapolação dos dados. Na maioria dos países a pesquisa ainda é insipiente.

Outro problema é a origem dos dados. As pesquisas sobre a SAl têm oferecido, fundamentalmente, evidências indiretas (O'FLAHERTY; PHILLIPS, 2015). Como fonte privilegiada estão os estudantes que vivenciam a SAl, que são, então, monitorados quanto ao seu desempenho ou questionados quanto as suas percepções (LONG; CUMMINS; WAUGH, 2016). Sabe-se, no entanto, que essa fonte é limitada e pode não ser segura. Delozier e Rhodes (2017) citam uma série de estudos metacognitivos para demonstrar que a percepção dos estudantes nem sempre é uma métrica adequada.

Uma nova questão é que a maior parte dos trabalhos consiste em pesquisas experimentais de comparação entre o modelo tradicional e o invertido. Os dados costumam ser coletados e analisados segundo abordagens quantitativas, com tratamentos estatísticos de comparação e correlação. Os instrumentos, no entanto, são quase que exclusivamente as surveys e testes de desempenho não padronizados, criados pelos próprios autores dos estudos (39\% e $28 \%$, respectivamente, de acordo com Zainuddin e Halili (2016)). Somente um estudo longitudinal aparece nas revisões de literatura.

As revisões ainda sugerem carências de rigor e objetividade ao se estabelecer métricas de aprendizagem, talvez pela ausência de referenciais teóricos de análise (outro fato comum) ou pela grande diversidade das iniciativas investigadas (BISHOP; VERLEGER, 2013; ABEYSEKERA; DAWSON, 2014; GIANNAKOS; KROGSTIE; CHRISOCHOIDES, 2014; O'FLAHERTY; PHILLIPS, 2015; HUBER; WERNER, 2016).

Nesse sentido, há também pouca evidência emergindo de pesquisas qualitativas a partir de abordagens críticas e fenomenológicas que valorizem peculiaridades de contextos e níveis de ensino, e/ou que tomem a prática docente ou a cultura institucional fontes de dados (ABEYSEKERA; DAWSON, 2014; ZAINUDDIN; HALILI, 2016). Karabulut-IIgu, Jaramillo Cherrez e Jahren (2017) acrescentam que o que se tem feito é, fundamentalmente, documentar o processo e compartilhar experiências, enquanto pesquisas de sustentação teórica e métodos de avaliação são, ainda, necessárias para estabelecer a pedagogia que ampara a SAI.

O'Flaherty e Phillips (2015) não hesitam ao afirmar a escassez de evidências conclusivas e sólidas sobre as contribuições da SAl, sobretudo ao que se convencionou chamar e defender de "aprendizagem ao longo da vida". 


\section{Crítica 5: Resultados divergentes}

Como exposto na seção introdutória, os resultados dos estudos sobre o desempenho, a frequência, o engajamento e a interação de estudantes em SAls são provocantes e promissores. A literatura, no entanto, vem mostrando que os resultados podem estar inflacionados e que os atribuir de maneira ampla e abrangente ao modelo SAI seria precipitado ou até equivocado.

A chefe do Departamento de Física em Harvard, por exemplo, encara com ceticismo a adoção da SAI. Embora Melissa Franklin elogie a já citada experiência do professor Eric Mazur, ela se mostra preocupada com relatos de estudantes que se sentem mal com as exigências do curso, e o fato de que isso repercuta nas más avaliações que fazem das disciplinas invertidas (BERRETT, 2012). No Brasil, experiências, como a de Suhr (2016), têm apontado que a falta de uma cultura de comprometimento dos estudantes é um dos principais desafios para a adoção bem-sucedida do modelo SAI.

Em um trabalho de ampla repercussão no campo, Strayer (2012) sustenta que, mesmo quando os estudantes se mostram mais responsáveis por sua aprendizagem e mais abertos ao trabalho cooperativo e criativo, eles se sentem menos satisfeitos com a estrutura da aula e a desorientação sobre as tarefas propostas. Para o autor, talvez a SAI não seja um modelo adequado para cursos introdutórios se comparado com classes mais avançadas, quando os estudantes estão mais interessados e dispostos a investigar os temas em estudo.

Recentemente, resultados encontrados por Jensen, Kummer e Godoy (2015) e DeLozier e Rhodes (2017) sugerem que os benefícios da SAI não decorrem necessariamente da "inversão" e do estudo prévio, mas sobretudo das características de aprendizagem ativa do encontro presencial. A acolhida dos estudantes à SAI teria uma relação mais íntima com as características da aula presencial (aí sim, associadas ao paradigma construtivista) do que com o estudo prévio e o contato com as aulas em vídeo.

Dois outros trabalhos endossam essa conclusão: o de Mennella (2016), que compara o desempenho e a satisfação de estudantes cursando uma disciplina de Genética em uma SAI com uma classe "convencional", mas que integra elementos da chamada aprendizagem ativa; e o de Sletten (2017), que examina a percepção dos estudantes sobre a SAl e o comprometimento com os estudos fora dela. Ambos demonstram não haver correlação direta do estudo prévio com a qualidade na experiência de aprendizagem ou com o desempenho. No caso de Sletten, a autora ainda apresenta dados que questionam a capacidade de autogerência do aprendizado pelos estudantes fora de sala.

Em outro estudo, com mais de 500 estudantes de Graduação e Pós-Graduação, McNally et al. (2017) sugerem haver dois grupos de estudantes: os que endossam a SAl e, consequentemente, demonstram atitudes positivas em relação ao modelo; e um grupo neutro para a maioria dos aspectos do modelo, mas muito resistente ao estudo prévio. 
Vários trabalhos mostram também não haver correlação entre percepções positivas para com a SAI e o desempenho de estudantes (FITZGERALD; LI, 2015; HOTLE; GARROW, 2015; HEYBORNE; PERRETT, 2016). Um deles, inclusive, é enfático em afirmar que, para os estudantes de alto desempenho, não faz diferença se a sala de aula é tradicional ou invertida (HOTLE; GARROW, 2015).

Consoante Moffett (2015), as decisões sobre o arranjo de qualquer disciplina devem se basear nas teorias educacionais e nas evidências da prática referendada pelos educadores, evitando o deslumbramento provocado pelas tecnologias educativas. A literatura desenvolvida até aqui remete à importância das peculiaridades e singularidades de cada contexto (área de conhecimento ou características dos estudantes, por exemplo), de modo que valem as experiências vicárias, mas se torna descabida a generalização dos modelos prontos (BISHOP; VERLEGER, 2013; ABEYSEKERA; DAWSON, 2014).

\section{Crítica 6: Riscos didáticos}

Conforme sugerido até aqui, a SAI não é um procedimento metodológico, mas um arranjo didático em um sentido mais amplo. As alterações que ela promove alcançam todos os elementos da didática e reconfiguram o sistema de ensino no qual se inserem, merecendo descrições e análises mais cuidadosas do que a literatura tem conseguido oferecer.

Críticos como Stager (2013) e Bogost (2013) apontam diretamente para o currículo. Segundo eles, o fenômeno da SAl é uma resposta burocrática aos currículos inchados, que não mais cabem no tempo didático para o qual foram desenvolvidos. Ao invés de repensar os currículos e o processo educativo como um todo, estar-se-ia optando por uma solução técnica (provavelmente ineficaz e antidemocrática) ao migrar o processo de ensino (e seus conteúdos) do tempo e espaço escolar institucionalizado para a vida privada do estudante. Para Bogost (2013), trata-se de uma transformação estrutural dirigida a tornar os encontros educativos institucionalizados, fundamentalmente avaliativos, enquanto se exacerba a responsabilidade do cidadão por sua formação.

Outro aspecto relevante é o risco de condensação dos conteúdos de ensino em uma proposta prêt-à-porte. Para Bogost (2013), a SAl estimula que os professores resumam suas aulas em formatos mais curtos, menos detalhados, mais adequados ao entretenimento ou à visão geral do conteúdo. Isso poderia afastar os estudantes do contato direto com os saberes construídos culturalmente, inclusive com os originais, e tornar ainda mais desafiador seu domínio pleno e genuíno dos conhecimentos propostos.

Em relação aos estudantes, há abundantes indícios na literatura sobre os riscos impostos de uma adoção açodada ou desatenta da SAI. Autores, em diferentes contextos - Al-Zahrani (2015), na Arábia Saudita, Pavanelo e Lima (2017), no Brasil e Sletten (2017), nos Estados Unidos -, relatam as dificuldades dos estudantes em se adaptar ao modelo ou em autorregular ou autogerenciar sua experiência de aprendizagem. A responsabilização e as exigências do processo são amplamente apontadas como desafios nos relatos de experiências. Além disso, identificam-se, ainda, dificuldades maiores para os estudantes menos preparados, que estariam mais susceptíveis a falhar e/ou abandonar a experiência - em um processo flagrantemente antidemocrático (CHING; BASHAM; 
JANG, 2005). A ampliação do tempo que os jovens passam em frente às telas (screen time) e o aumento da carga de trabalho extraclasse (JOHNSON, 2013; WAGNER; LAFORGE; CRIPPS, 2013), também são pontuados, rememorando os inquietantes questionamentos propostos de Kohn (2006), na obra "O mito do dever de casa".

Considerando a docência, os desafios também são amplos. Wagner, Laforge e Cripps (2013) destacam como uma das principais dificuldades discutidas pela literatura o ônus, em tempo e esforço de trabalho, na reestruturação de todo o material de uma disciplina para que ela se viabilize no modelo invertido. Experiências, como a relatada por Pavanelo e Lima (2017), expõem as dificuldades com o acervo de materiais adequados à SAI. Inclusive, já se observa autores percebendo o desafio e propondo "manuais" para impedir que a SAI fracasse ("To prevent flip from flopping", em um trocadilho divertido); tudo porque a práxis docente é alcançada pela mudança e, como já foi dito, o professor precisaria partilhar os pressupostos pedagógicos da SAI para ter sucesso (O'FLAHERTY; PHILIPS, 2015). As metodologias, as posturas, as ações, os juízos, os critérios, as deliberações e as avaliações que os docentes fazem/tomam não podem conflitar com o projeto pedagógico do modelo, sob pena de que ele não se efetive como planejado. As dificuldades, portanto, são tantas e profundas que muitos professores acabam desistindo depois das primeiras experiências (RODRIGUES, 2015).

Por fim, alguns autores defendem algo ainda mais severo: que a SAI não promoveria uma modernização pedagógica da prática docente, mas possivelmente a descaracterizaria. Segundo Stager (2013), o modelo invertido, dependendo de sua estrutura metodológica, deprecia a importância de professores experimentados e bem-formados, tornando possível substituí-los por executores técnicos ou tutores (focados no funcionamento das metodologias e na avaliação da atividade dos estudantes).

\section{Crítica 7: Interesses não pedagógicos}

Já se percebeu que a modernização dos processos de ensino-aprendizagem, ensejada pelo modelo SAl, carrega em seu bojo pautas que transcendem as dimensões pedagógica e didática. Ao reivindicar uma educação híbrida (blended learning), a SAI enceta novos mercados para a produção e publicação de conteúdos de ensino. Embora haja iniciativas públicas e institucionais, como repositórios de recursos educacionais abertos e cursos massivos on-line (MOOCs, na sigla em inglês), vem da iniciativa privada o grande volume de softwares, plataformas e repositórios dos conteúdos de ensino. São ferramentas para criar apresentações, questionários interativos, murais virtuais, atividades individuais ou colaborativas e, inclusive, avaliar os trabalhos dos estudantes.

Sem discutir o mérito acadêmico desses produtos e serviços, artigos de opinião, como os de Stager (2011) e Pacheco (2014), alimentam a preocupação com a repercussão desses poderosos interesses econômicos sobre as políticas educacionais. Sua desconfiança aponta para os interesses mercadológicos, por exemplo, das grandes plataformas de distribuição digital de vídeos. Essas empresas "monetizam" visualizações e associam contratos comerciais publicitários ao seu conteúdo, de modo que seria altamente rentável defender e promover um cenário educativo em que todo estudante consuma compulsoriamente conteúdos na rede web. 
Pacheco (2014) fala em "colonialismo pedagógico" e acusa a receptividade da pedagogia brasileira à SAI de constrangedora. Stager (2013) salienta que desinstitucionalizar o ensino não é apenas inadequado, mas antidemocrático. Bogost (2013) propõe que um ensino híbrido, condensado, resumido, que responsabiliza mais o estudante e menos a instituição, é muito adequado aos interesses de empresas e governos que querem a redução de custos da oferta educacional. Este autor salienta que "Não é por acaso que empresas privadas de $\mathrm{MOOC}$, como a Coursera, defendem salas de aula invertidas, uma vez que essas organizações têm muito a ganhar com o seu endosso pelas universidades" (2013. p. 1). Ilustrando a acusação, vale registrar que esta mesma empresa produziu um guia de campo para a instalação da SAl, documento que veicula as experiências de universidades como Stanford, Duke e Valderbilt, nos Estados Unidos, e faz uma defesa enfática da adoção do modelo. Ainda naquele país, uma das maiores empresas do mundo no mercado editorial, a Pearson, é dona do registro da marca F-L-I-P - acrônimo para a expressão "Flexible Environment, Learning Culture, Intentional Content and Professional Educator", expressões amplamente divulgadas como os pilares dessa mudança educacional.

As preocupações sobre a mercantilização de serviços e produtos educacionais não são novas (GIBBONS, 1982 apud ENT, 2016), mas as acusações merecem aprofundamento teórico e empírico. Essas questões são legítimas e deveriam ocupar professores, pesquisadores e gestores da educação alvejados a todo o momento por cursos, guias e manuais de orientação e execução amparados ou desenvolvidos a partir de fontes altamente interessadas. Abeysekera e Dawson (2014) sugerem o risco de que, no lugar de uma política educacional baseada em evidências, estejamos nos sujeitando a "evidências baseadas em políticas". O ceticismo e a vigilância crítica são virtudes em um contexto no qual as propostas neoliberais alcançam, de modo fulminante, a educação.

\section{EM SÍNTESE}

Sim, a SAl é um fenômeno educativo contemporâneo! A proposta de ensino prévio extraclasse, mediado por tecnologias digitais e seguido por metodologias ativas em sala, atinge a educação básica e o ensino superior em todo o globo. Diversos saberes produzidos pelas ciências da educação são postos em debate e todas as dimensões didáticas - epistemológica (conhecimento), psicológica (estudantes) e praxeológica (professores) - do ensino formal são alcançadas. Resultados alvissareiros se acumulam, repercutem entre a comunidade de educadores e induzem políticas públicas e institucionais. Questionamentos pedagógicos, sociológicos, metodológicos e empíricos, no entanto, também existem, formalizados e documentados, e não podem ser negligenciados ou escamoteados.

A SAI, por si, não configura panaceia pedagógica ou revolução tecnológica. Seu potencial transformador depende do discernimento e do juízo emanado de uma educação socialmente referenciada, de uma pesquisa acadêmica rigorosa e de uma prática docente baseada em evidências. 
As críticas elencadas neste artigo não negam o potencial pedagógico e didático dessa abordagem, mas ilustram a necessidade de melhores e mais profundas reflexões sobre o tema, enquanto indicam outros caminhos possíveis para os esforços de pesquisas e as práticas educativas.

\section{REFERÊNCIAS}

ABEYSEKERA, L.; DAWSON, P. Motivation and cognitive load in the flipped classroom: definition, rationale and a call for research. Higher Education Research \& Development, p. 1-14, set. 2014.

AL-ZAHRANI, A. M. From passive to active: The impact of the flipped classroom through social learning platforms on higher education students' creative thinking. British Journal of Educational Technology, vol. 46, n. 6, p. 1.133-1.148, 2015.

ATWOOD, G. S. How to Prevent Your Flip from Flopping: Five Key Mistakes to Avoid When Switching to the Flipped Classroom Model. University Libraries Faculty and Staff Publications. Paper 43. 2016. Disponível em: <http://scholarworks.uvm.edu/libfacpub/43>. Acesso em: 10 set. 2017.

AVELLAR, S. A sala de aula com novos formatos em tempos digitais. O Globo, Rio de Janeiro, 4 mar. 2013. Disponível em: <https://oglobo.globo.com/sociedade/educacao/a-sala-de-aula-com-novos-formatos-em-tempos-digitais-7721124\#ixzz4uNTFy016>. Acesso em: 11 set. 2017.

BARROWS, H. S.; TAMBLYN R. M. Problem-based learning: an approach to medical education. New York: Springer, 1980.

BART, M. Survey confirms the growth of the flipped classroom. Faculty Focus. 2013. Disponivel em: <https://www.facultyfocus.com/articles/blended-flipped-learning/survey-confirms-growth-of-the-flipped-classroom/>. Acesso em: 20 out. 2017.

BASTOS FILHO, J. B. Uma controvérsia em torno da educação científica: partidários e críticos do construtivismo. Caderno Brasileiro de Ensino de Física, v. 32, n. 2, p. 299-319, 2015.

BERBEL, N. A. N. As metodologias ativas e a promoção da autonomia de estudantes. Semina: Ciências Sociais e Humanas, Londrina, v. 32, n. 1, p. 25-40, jan./jun. 2011. Disponível em: <http://www.uel.br/ revistas/uel/index.php/seminasoc/article/view/10326/10999>. Acesso em: 12 ago. 2016.

BERGMANN, J.; SAMS, A. Flip Your Classroom: reach every student in every class every day. Eugene, Oregon: Iste, 2012.

BERRETT, D. How flipping the classroom can improve the traditional lecture. Chronicle of Higher Education, 19, fev. 2012.

BIBANO, B. Salas de aula invertidas, uso de impressora 3D e outras tendências do Ensino Superior. Veja, São Paulo, 9 mar. 2014. Disponível em: <http://veja.abril.com.br/educacao/salas-de-aula-invertidas-uso-de-impressora-3d-e-outras-tendencias-do-ensino-superior/>. Acesso em: 11 set. 2017.

BISHOP, J. L.; VERLEGER, M. A. The Flipped Classroom: A Survey of the Research. Proccedings of the Annual Conference of the American Society for Engineering Education, jan. 2013. p. 6.219.

BOGOST, I. The Condensed Classroom: "Flipped" classrooms don't invert traditional learning so much as abstract it. The Atlantic, 27 ago. 2013. Disponível em: <https://www.theatlantic.com/technology/archive/2013/08/the-condensed-classroom/279013/>. Acesso em: 15 abr. 2013.

BROWN, A. F. A phenomenological study of undergraduate instructors using the inverted or flipped classroom model. 2012. Dissertation (Doctor of Education) - Pepperdine University, 2012. Disponível em: <http://pepperdine.contentdm.oclc.org/cdm/ref/collection/p15093coll2/id/348>. Acesso em: 11 set. 2017.

BUSATO, P. et al. Student performance in conventional and flipped classroom learning environments. Applied Engineering in Agriculture, v. 32, n. 5, 2016.

CASTELLO BRANCO, C. et al. A sala de aula invertida como metodologia convergente ao paradigma da complexidade. Boletim Técnico do Senac, v. 43, n. 2, 2016.

CARVALHO, A. M. P. O ensino de ciências e a proposição de sequências de ensino investigativas. In: CARVALHO, A. M. P. (Org.). Ensino de Ciências por investigação: condições para implementação em sala de aula. São Paulo: Cengage Learning, 2013, p. 1-20. Cap. 1.

CHING, C. C.; BASHAM, J. D.; JANG, E. The legacy of the digital divide: gender, socioeconomic status, and early exposure as predictors of full-spectrum technology use among young adults. Urban Education, 40 (4), p. 394-411, 2005. 
DELOZIER, S. J.; RHODES, M. G. Flipped Classrooms: a Review of Key Ideas and Recommendations for Practice. Educational Psychology Review, v. 29, n. 1, p. 141-151, 2017.

DESLAURIERS, L.; SCHELEW, E.; WIEMAN, C. Improved learning in a large-enrollment physics class. Science, n. 332, p. 862-864, 2011.

DRISCOLL, T. Flipped Learning and democratic Education: The Complete Report. 2012. Disponível em: <http://www.flipped-history.com/2012/12/flipped-learning-democratic-education.html>. Acesso em: 1 은 mar. 2016.

ENT, V. I. Is flipped learning really new to academia? TechTrends, n. 60, p. 204-206, 2016.

FITZGERALD, N.; LI, L. Using presentation software to flip an undergraduate analytical chemistry course. Journal of Chemical Education, 92(9), p. 1.559-1.563, 2015.

FITZPATRICK, M. Classroom lectures go digital. The New York Times, (24), jun. 2012.

FUNDAÇÃO GETÚLIO VARGAS (FGV). Sala de aula invertida. Revista Ei! Ensino Inovativo, Escola de Administração de Empresas de São Paulo, v. 2, p. 14-17, 2015.

GALINDO, J. J.; QUINTANA, M. G. B. Innovación docente a través de la metodología flipped classroom: percepción de docentes y estudiantes de educación secundaria.Didasc@lia: Didáctica y Educación, v. VII, p. 153-172, 2016.

GIANNAKOS, M. N.; KROGSTIE, J.; CHRISOCHOIDES, N. Reviewing the flipped classroom research. Proceedings of the Computer Science Education Research Conference on - CSERC', 14, p. 23-29, fev. 2014.

GÓMEZ, A. G. Aprendizaje inverso y motivación en el aula universitaria. Pulso, v. 39, p. 199-218, 2016.

HEYBORNE, W.; PERRETT, J. To flip or not to flip? Analysis of a flipped classroom pedagogy in a general biology course. Journal of College Science Teaching, 45(4), p. 31-37, 2016.

HOTLE, S.; GARROW, L. Effects of the traditional and Flipped classrooms on undergraduate student opinions and success. Journal of Professional Issues in Engineering Education and Practice, 142(1), 2015.

HUBER, E.; WERNER, A. A review of the literature on flipping the STEM classroom: preliminary findings. Show Me The Learning. Proceedings Ascilite. Adelaide: Ascilite, 2016.

INOVAÇÃO NA EDUCAÇÃO: como usar as novidades mais promissoras em sua sala de aula. Revista Nova Escola, ano 32, n. 299, fev. 2017.

JENSEN, J. L.; KUMMER, T. A.; GODOY, P. D. D. M. Improvements from a flipped classroom may simply be the fruits of active learning. CBE - Life Sciences Education, vol. 14, p. 1-12, spring 2015.

JOHNSON, G. B. Student perceptions of the flipped classroom. 2013. MA thesis - University of British Columbia, Canadá, 2013.

JOHNSON, L. et al. NMC Horizon Report: 2014. Higher Education Edition. Austin, Texas: The New Media Consortium, 2014.

KARABULUT-ILGU, A.; JARAMILLO CHERREZ, N.; JAHREN, C. T. A systematic review of research on the flipped learning method in engineering education. British Journal of Educational Technology, 2017.

KIM, K. Y.; KIM, Y. What are learning satisfaction factors in flipped learning? Advances in Computer Science and Ubiquitous Computing. CSA 2016, CUTE 2016, UCAWSN 2016. Lecture Notes in Electrical Engineering, vol. 421, 2017.

KOHN, A. The homework myth: why our kids get too much of a bad thing. Cambridge, MA: Da Capo Life Long, 2006.

LAGE, M. J.; PLATT, G. J.; TREGLIA, M. Inverting the classroom: a gateway to creating an inclusive learning environment. Journal of Economic Education, 3(1), p. 30-43, 2000.

LONG, T.; CUMMINS, J.; WAUGH, M. Use of the flipped classroom instructional model in higher education: instructors' perspectives. Journal of Computing in Higher Education, p. 1-22, 2016.

LYMAN, F. Think-Pair Share: An expanding teaching technique: MMA-CIE. Cooperative News, v. 1, p. 1-2, 1987.

MAIA, D. Na sala de aula invertida, alunos antecipam conteúdo em casa. Folha de São Paulo, São Paulo, 11 set. 2016. Disponível em: <http://www1.folha.uol.com.br/educacao/2016/09/1811790-na-sala-de-aula-invertida-alunos-antecipam-conteudo-em-casa.shtml>. Acesso em: 11 set. 2017.

MANGAN, K. Inside the flipped classroom. Chronicle of Higher Education, 30, set. 2013. Disponível em: <http://www.chronicle.com/article/A-Guide-to-the-Flipped/151039>. Acesso em: 10 mar. 2016.

MARLOWE, C. A. The Effect of the Flipped Classroom On Student Achievement and stress. 2012. Master's thesis - Montana State University, 2012. Disponível em: <http://scholarworks.montana.edu/xmlui/bitstream/handle/1/1790/MarloweC0812.pdf?sequence=1>. Acesso em: 15 mar. 2015. 
MASCOLO, M. F. Beyond student-centered and teacher-centered pedagogy: teaching and learning as guided participation. Pedagogy and the Human Sciences, vol. 1, n. 1, p. 3-27, 2009.

MATTHEWS, M. Construtivismo e o ensino de ciências: uma avaliação. Cad. Cat. Ens. Fís., v. 17, n. 3, p. 270-294, dez. 2000.

MAZUR, E. Peer Instruction: a user's manual. Upper Saddle River: Prentice Hall, 1997. Farewell, Lecture? Science, v. 323, p. 50-51, 2009.

MCNALLY, B. et al. Flipped classroom experiences: student preferences and flip strategy in a higher education context. Higher Education, v. 73, n. 2, p. 281-298, 2017.

MENNELLA, T. A. Comparing the efficacy of flipped vs. alternative active learning in a college genetics course. The American Biology Teacher, v. 78, n. 6, p. 471-479, 2016.

MICHAEL, J. Where's the evidence that active learning works? Advances Physiology Education, n. 30, p. 159-167, 2006.

MICHAELSEN, L. K.; KNIGHT, A. B.; FINK, L. D. Team-based learning: a transformative use of small groups in college teaching. Sterling. VA: Stylus Publishing, LLC, 2004.

MOFFETT, J. Twelve tips for "flipping" the classroom. Medical Teacher, 37:4, p. 331-336, 2015.

MORAN, J. Mudando a educação com metodologias ativas. In: SOUZA, C. A. de; MORALES, O. E. T. (Org.). Convergências midiáticas, educação e cidadania: aproximações jovens. Ponta Grossa: Foca Foto-Proex/ UEPG, 2015. Vol. II. (Coleção mídias contemporâneas).

MORENA COSTA, M. Sala de aula invertida faz alunos aprenderem de forma livre. Porvir, 3 maio 2016. Disponível em: <http://porvir.org/sala-de-aula-invertida-faz-os-alunos-aprenderem-de-forma-livre/>. Acesso em: 11 set. 2017.

MÜLLER, M. G. et al. Uma revisão da literatura acerca da implementação da metodologia interativa de ensino peer instruction (1991 a 2015). Revista Brasileira de Ensino de Física, v. 39, n. 3, p. e3.403, 2017.

NESHYBA, S. It's a flipping revolution. Chronicle of Higher Education, n. 4 abr. 2013. Disponível em: <http://www.chronicle.com/article/A-Guide-to-the-Flipped/151039>. Acesso em: 10 mar. 2016.

NOURI, J. The flipped classroom: for active, effective and increased learning - especially for low achievers. International Journal of Educational Technology in Higher Education, v. 13, n. 1, p. 1-10, 2016.

NOVAK, G. M. et al. Just-in-time teaching: blending active learning with web technology. Upper Saddle River: Prentice Hall, 1999.

O'FLAHERTY, J.; PHILLIPS, C. The use of flipped classrooms in higher education: a scoping review. Internet and Higher Education, v. 25, p. 85-95, 2015.

OLAKANMI, E. E. The Effects of a Flipped Classroom Model of Instruction on Students??? Performance and Attitudes Towards Chemistry. Journal of Science Education and Technology, p. 1-11, 2016.

OFUGI, M. S. A sala de aula invertida como técnica alternativa de ensino: um enfoque no desenvolvimento da autonomia do aprendiz de inglês como L2/LE. 2016. Dissertação (Mestrado) - Universidade Federal de Goiás, Faculdade de Letras (FL), Programa de Pós-Graduação em Letras e Linguística, Goiânia, 2016.

PACHECO, J. Sala de aula invertida: por que não reagem os pedagogos brasileiros ao neocolonialismo pedagógico? Revista Educação, n. 5, maio 2014. Disponível em: <http://www.revistaeducacao.com.br/ sala-de-aula-invertida/>. Acesso em: 1으. 2016.

PAPADOPOULOS, C.; ROMAN, A. S. Implementing an inverted classroom model in engineering statics: Initial results. Proceedings of the 40th ASEE/IEEE Frontiers in Education Conference. Washington DC: American Society for Engineering Statistics, 2010.

PAVANELO, E.; LIMA, R. Sala de aula invertida: a análise de uma experiência na disciplina de Cálculo I. Bolema, Rio Claro, SP, v. 31, n. 58, p. 739-759, ago. 2017.

PIVA, N. Método da "sala de aula invertida" troca os papéis de casa e da escola. Gazeta do Povo, Curitiba, 11 jan. 2016. Disponível em: <http://www.gazetadopovo.com.br/educacao/metodo-da-sala-de-aula-invertida-troca-os-papeis-de-casa-e-da-escola-0jnv8isjepw6p94pvm92thgwm>. Acesso em: 11 set. 2017.

ROCHA, H. M.; LEMOS, W. M. Metodologias ativas: do que estamos falando? Base conceitual e relato de pesquisa em andamento. SIMPÓSIO PEDAGÓGICO E PESQUISAS EM COMUNICAÇÃO, 9., 2014, [Resende - RJ].

RODRIGUES, C. S. Aula invertida: desafios de uma nova metodologia e um novo olhar do professor. 2015. Dissertação (Mestrado em Educação) - PUC/PR, Curitiba, 2015.

SENGEL, E. To FLIP or not to FLIP: Comparative case study in higher education in Turkey. Computers in Human Behavior, v. 64, p. 547-555, 2016.

SLETTEN, S. R. Investigating Flipped Learning: Student Self-Regulated Learning, Perceptions and Achievement in an Introductory Biology Course. Journal of Science Education and Technology, n. 26, p. 347, 2017. 
STAGER, G. Can flipping the classroom fix the educational system? Entrevista concedida à Southern California Public Radio. AirTalk [podcast]. fev. 2013.

Who elected Bill Gates? Huffington Post. 2011. Disponível em: <http://www.huffingtonpost.com/ gary-stager/who-elected-bill-gates_b_829456.html>. Acesso em: 10 mar. 2016.

STRAYER, J. How learning in an inverted classroom influences cooperation, innovation and task Orientation. Learning Environments, 15(2), p. 171, 2012.

SUHR, I. R. F. Desafios no uso da sala de aula invertida no Ensino Superior. Transmutare, v. 1, n. 1, 2016.

TALBERT, R. Inverting the Linear Algebra Classroom. Primus, 24(5), p. 361-374, 2014.

TAZIJAN, F. N. et al. Building communication skills through flipped classroom. International Academic Research Journal of Social Science, 3(1), p. 142-147, 2017.

TENENBAUM, G. et al. Constructivist pedagogy in conventional on - campus and distance learning practice: an exploratory investigation. Learning and Instruction, n. 11, p. 87-111, 2001.

TREVELIN, A. T. C.; PEREIRA, M. A. A.; OLIVEIRA NETO, J. D. de. A utilização da "sala de aula invertida" em cursos superiores de tecnologia: comparação entre o modelo tradicional e o modelo invertido "flipped classroom" adaptado aos estilos de aprendizagem. Revista de Estilos de Aprendizagem, Madrid, v. 11, n. 12, p. 137-150, out. 2013.

TURAN, Z.; GOKTAS, Y. The flipped classroom: Instructional efficiency and impact on achievement and cognitive load levels. Journal of E-Learning and Knowledge Society, v. 12, n. 4, p. 51-62, 2016.

VALENTE, J. A. Blended learning e as mudanças no Ensino Superior: a proposta da sala invertida. Educar em Revista, Curitiba, Brasil, Edição Especial, n. 4, p. 79-97, 2014.

VILLAS BÔAS, M. A. Aulas invertidas são muito mais eficientes e inclusivas. Carta Capital, São Paulo, 25 ago. 2017. Disponível em: <https://www.cartacapital.com.br/blogs/vanguardas-do-conhecimento/aulas-invertidas-sao-muito-mais-eficientes>. Acesso em: 11 set. 2017.

WAGNER, D.; LAFORGE, P.; CRIPPS, D. Lecture material retention: A first trial report on flipped classroom strategies in electronic systems engineering at the University of Regina. Conference Proceedings of the Canadian Engineering Education Association, Montreal, Canadá, june 2013.

YOUNG, J. When a flipped-classroom pioneer hands off his video lectures, this is what happens. Chronicle of Higher Education, n. 7, jan. 2015. Disponível em: <http://www.chronicle.com/article/A-Guide-to-theFlipped/151039>. Acesso em: 1으. 2016.

ZAINUDDIN, Z.; HALILI, S. H. Flipped Classroom Research and Trends from Different Fields of Study. International Review of Research in Open and Distributed Learning, v. 17, n. 3, p. 313-340, 2016. 